\title{
Mild steel welding is associated with alterations in circulating levels of cancer-related proteins
}

\author{
Anda R. Gliga ${ }^{1} \cdot$ Tahir Taj $^{2} \cdot$ Maria Hedmer² $\cdot$ Eva Assarsson ${ }^{2} \cdot$ Lars Rylander² $^{2}$ Maria Albin ${ }^{1,2} \cdot$ Karin Broberg $^{1,2}$ (I)
}

Received: 26 July 2019 / Accepted: 8 October 2019 / Published online: 22 October 2019

(c) The Author(s) 2019

\begin{abstract}
Welding fumes were recently classified as carcinogenic to humans and worldwide millions work as welders or perform welding operations. The purpose of this study was to identify new biomarkers of welding-induced carcinogenesis. We evaluated a panel of 91 putative cancer-related proteins in serum in a cohort of welders working with mild steel $(n=77)$ and controls $(n=94)$ from southern Sweden sampled on two occasions 6-year apart using a longitudinal analysis (linear mixed models). The significant results from the longitudinal analysis were tested for reproducibility in welders $(n=88)$ and controls $(n=69)$ sampled once during the same sampling period as timepoint 1 or timepoint 2 (linear regression models), i.e., in a cross-sectional setting. The models were adjusted for age, body-mass index, and use of snus. All study participants were non-smokers at recruitment. Exposure to welding fumes was assessed using questionnaires and respirable dust measurement in the breathing zone that was adjusted for personal respiratory protection equipment. The median respirable dust in welders was $0.7(0.2-4.2)$ and $0.5(0.1-1.9) \mathrm{mg} / \mathrm{m}^{3}$ at the first and second timepoints, respectively. We identified 14 cancer-related proteins that were differentially expressed in welders versus controls in the longitudinal analysis, out of which three were also differentially expressed in the cross-sectional analysis (cross-sectional group). Namely, syndecan 1 (SDC1), folate receptor 1 (FOLR1), and secreted protein acidic and cysteine rich (SPARC) were downregulated, in welders compared with controls. In addition, FOLR1 was negatively associated with years welding. Disease and function analysis indicated that the top proteins are related to lung cancer as well as cell invasion and migration. Our study indicates that moderate exposure to welding fumes is associated with changes in circulating levels of putative cancer-related proteins, out of which FOLR1 showed a clear dose-response relationship. It is, however, unclear to which extent these changes are adaptive or potential early biomarkers of cancer.
\end{abstract}

Keywords Welding $\cdot$ Biomarkers $\cdot$ Cancer $\cdot$ Particles $\cdot$ Occupational $\cdot$ SDC1 $\cdot$ FOLR1 $\cdot$ SPARC

Electronic supplementary material The online version of this article (https://doi.org/10.1007/s00204-019-02594-4) contains supplementary material, which is available to authorized users.

Karin Broberg

karin.broberg@ki.se

Anda R. Gliga

anda.gliga@ki.se

Tahir Taj

tahir.taj@med.lu.se

Maria Hedmer

maria.hedmer@med.lu.se

Eva Assarsson

eva.assarsson@med.lu.se

\section{Background}

Worldwide, there are around 11 million welders and 110 million additional workers exposed to welding fumes (Guha et al. 2017). In Sweden, there are around 25,000 welders and

Lars Rylander

lars.rylander@med.lu.se

Maria Albin

maria.albin@ki.se

1 Institute of Environmental Medicine, Karolinska Institutet, Stockholm, Sweden

2 Division of Occupational and Environmental Medicine, Lund University, Lund, Sweden 
200,000-250,000 additional workers that perform welding along with their main work task (Sjögren 2013). Welding results in exposure to high levels of fine- and ultrafine welding particles, UV radiation, and, in some cases, welders have co-exposure to asbestos and solvents (Guha et al. 2017). The composition of the welding fumes is highly dependent on the materials used for welding (e.g., mild or stainless steel electrodes) and the type of welding process (e.g., gas or arc welding).

The International Agency for Research on Cancer (IARC) recently classified welding fumes as Group 1, i.e., "carcinogenic to humans" (Guha et al. 2017; IARC 2019), based on epidemiological evidence showing increased risk of lung cancer in welders. The risk for lung cancer was higher in welders that had a longer or higher cumulative exposure (Matrat et al. 2016; Siew et al. 2008; Sorensen et al. 2007; $t$ Mannetje et al. 2012). The assessment is valid for welding fumes in general, i.e., it includes both mild- and stainless steel welding (IARC 2019) and the key carcinogenic components of welding fumes are considered to be the particles. Strategies to identify the cancer risk associated with exposure to welding fumes are, therefore, needed, and a sound approach would be to develop methods to measure both the cancer-relevant particles at the workplace as well as early biomarkers of welding-related lung cancer.

The mechanisms behind the carcinogenicity of welding fumes are not fully understood. Several studies reported, for example, an increase in oxidative stress markers (Han et al. 2005; Hoffmeyer et al. 2012a, b), systemic inflammation (Kim et al. 2005; Shen et al. 2018; Wang et al. 2005), and immune suppression (Grigg et al. 2017; Marongiu et al. 2016) following exposure to welding fumes. In contrast, in cross-sectional studies of Swedish welders and controls, we found limited evidence for increased oxidative stress and inflammation, by measuring 8-oxodG in urine as well as cytokines and CRP in the blood ( $\mathrm{Li}$ et al. $2015 \mathrm{a}, \mathrm{b})$. An in vivo study indicated that welding fumes derived from stainless steel welding act as a cancer promoter and lead to lung cancer in mice previously exposed to 3-methylcholanthrene, a tumor inducer (Zeidler-Erdely et al. 2013).

Other mechanisms of welding-induced cancer could involve interactions with the DNA, without preceding inflammation. In a cross-sectional approach, we found slightly shorter telomeres in welders compared with controls ( $\mathrm{Li}$ et al. 2015b). In the same study sample, we found that welders had lower DNA methylation (hypomethylation) of the F2RL3 gene (Hossain et al. 2015), which has been suggested to be a prospective marker for risk of lung cancer (Zhang et al. 2015).

The goal of this study is to identify early biomarkers for welding-related cancer risk. To this end, we evaluated a panel of 91 putative cancer-related proteins in a cohort of welders and controls from southern Sweden, sampled on two occasions 6-year apart. In addition, based on the results from the longitudinal data, the observed significant associations were further evaluated in welders and controls sampled once, i.e., in a cross-sectional setting (cross-sectional group; flowchart of the study participants in Fig. 1). The evaluated proteins are involved in processes critical for cancer initiation and progression such as cell adhesion, motility, proliferation, differentiation, angiogenesis, and immune response.

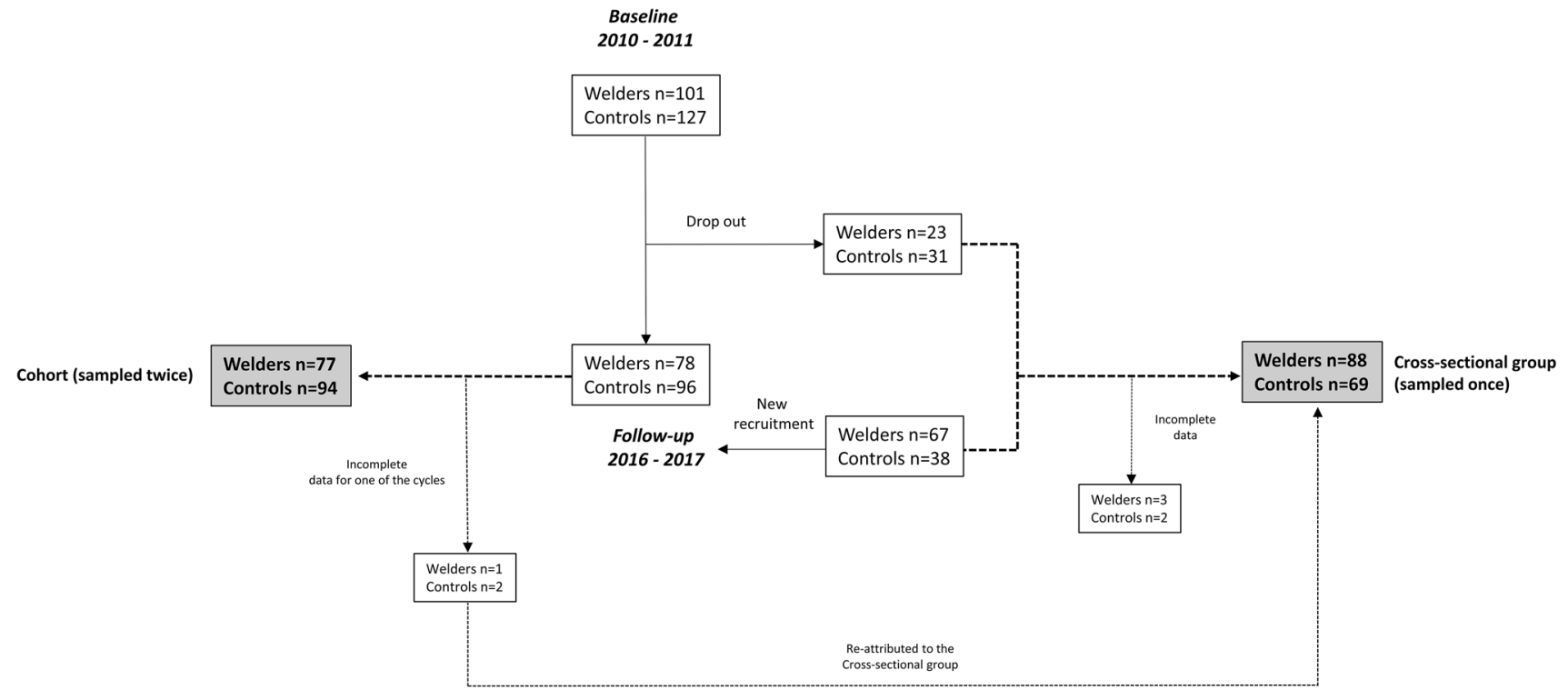

Fig. 1 Flowchart of the study design. Incomplete data refer to incomplete questionnaire and/or low quality of the protein data 


\section{Results}

\section{Characteristics of the study participants}

All study participants were males with a relatively healthy lifestyle: the majority were non-smokers, and had low alcohol consumption and a medium-high intake of vegetables (Table 1). A comparison analysis between welders and controls from the three study groups (main study cohort examined at two occasions-timepoint 1 and 2-and cross-sectional group examined once) revealed that welders were more likely to be born outside Sweden $(p<0.005)$ as well as to live in towns and countryside rather than in large or small cities $(p<0.005)$. There were no other significant differences between welders and controls as regards the evaluated characteristics. An additional comparison analysis between the three study groups revealed that age was significantly different $(p<0.001)$; age for the cross-sectional group was more similar with the age for cohort at timepoint 1 . There were no other significant differences between the main study cohort and the cross-sectional group.

Between the two sampling occasions, both the years of welding and the cumulative exposure increased significantly for welders at timepoint 2 compared with timepoint 1 (Table 1). There was no significant change in the respirable dust levels measured in the workplace $(1.1 \mathrm{mg} /$ $\mathrm{m}^{3}$ at timepoint 1 and $0.9 \mathrm{mg} / \mathrm{m}^{3}$ at timepoint 2). The median exposure to respirable dust among the welders (i.e., respirable dust adjusted for PPE-personal protection equipment) was slightly higher at timepoint $1\left(0.7 \mathrm{mg} / \mathrm{m}^{3}\right)$ compared with timepoint $2\left(0.5 \mathrm{mg} / \mathrm{m}^{3}\right)(p$ value $=0.052)$. The median area levels of respirable dust in the control companies were for timepoint 1 and timepoint $20.09 \mathrm{mg} /$ $\mathrm{m}^{3}(0.02-0.2)$ and $0.03 \mathrm{mg} / \mathrm{m}^{3}(0.02-0.06)$, respectively. The cross-sectional group of welders had a median respirable dust level of $1.6 \mathrm{mg} / \mathrm{m}^{3}\left(0.8 \mathrm{mg} / \mathrm{m}^{3}\right.$ when adjusted for PPE). The correlations between years of welding and respirable dust levels (adjusted PPE) were in general very low $\left(\mathrm{r}_{\mathrm{S}}=-0.07\right.$ for timepoint $1, \mathrm{r}_{\mathrm{S}}=-0.14$ for timepoint 2 and $r_{S}=-0.12$ for the cross-sectional group). Body-mass index increased significantly at timepoint 2 compared with timepoint 1 in both welders and the controls (Table 1). None of the other characteristics of the study participants changed significantly between the two sampling occasions.

Principal component analysis was performed to evaluate to which extent characteristics of the study participants could explain the variation in serum levels of cancer-related proteins. The complete description of the 91 measured proteins is appended in Supplementary Table 1. The three groups, cohort timepoint 1 and 2 and the cross-sectional group, showed similar patterns, with age, body-mass index, and use of snus being the most significant parameters explaining protein variation (Fig. 2). For this reason, age, body-mass index and use of snus were used as covariates in the statistical analyses. The use of snus explained to a large extent cornulin (CRNN) levels in serum; removing CRNN from the analysis reduced the impact of snus on explaining the overall variation in the data set (data not shown). The principal component analysis did not reveal any association with variables such as residence, personal history of cancer, or particle exposure from hobby activities.

\section{Differentially expressed cancer-related proteins in welders compared with controls}

Using a longitudinal analysis (linear mixed models), we identified 14 out of 91 serum proteins that were significantly different in welders compared with controls (Table 2). Syndecan 1 (SDC1) was also significant after adjusting for multiple testing. Complete output data from the differential expression analysis between welders and controls are appended in Supplementary Table 2. Sensitivity analyses were performed (1) only including the non-smokers $(n=74$ welders, $n=89$ controls) and (2) by excluding welders with respirable dust levels $>2.5 \mathrm{mg} / \mathrm{m}^{3}(n=10)$. All identified proteins in the linear mixed models were significant $(p$ value $<0.05)$, with the exception of KLK8 $(p$ value $=0.059)$ in the sensitivity analyses.

Three of the proteins identified by linear mixed models, i.e., syndecan 1 (SDC1), folate receptor 1 (FOLR1), and secreted protein acidic and cysteine rich (SPARC), were also differentially expressed in the replication group, and additionally, two proteins, i.e., kallikrein-related peptidase 8 (KLK8) and ephrin-receptor A2 (EPHA2), showed $p<0.1$ in the cross-sectional group. The majority of the significant proteins (12 out of 14) in the study cohort were altered in the same direction in the cross-sectional group (Table 2). SDC1, FOLR1, and SPARC were downregulated in welders as compared with controls in both the longitudinal and the cross-sectional analysis (Table 2, Fig. 3). In addition, FOLR 1 seemed to decrease from timepoint 1 to timepoint 2 both in welders and in controls (Fig. 3).

Disease and function analysis of the top 20 differentially expressed proteins $(p<0.1)$ indicated lung cancer as top disease and cell movement (cell migration and cell invasion) as top functions (Fig. 4). Based on our data set, cell migration and cell invasion were predicted to be inhibited in welders versus controls.

Next, we evaluated the association between the three differentially expressed proteins (SDC1, FOLR1, and SPARC identified by linear mixed models and linear models of welders versus controls) and assessment of exposure to welding, i.e., respirable dust $(n=56)$, years 
Table 1 Characteristics of the main study group (cohort measured twice) and cross-sectional group (measured once) of welders and controls

\begin{tabular}{|c|c|c|c|c|c|c|c|c|}
\hline & \multicolumn{2}{|l|}{ Cohort timepoint 1} & \multicolumn{2}{|c|}{ Cohort timepoint 2} & \multicolumn{2}{|c|}{ Cross-sectional group } & \multirow[t]{2}{*}{$p$ value $^{\mathrm{i}}$} & \multirow[t]{2}{*}{$p$ value $^{\mathrm{n}}$} \\
\hline & $\begin{array}{l}\text { Welders } \\
(n=77)\end{array}$ & $\begin{array}{l}\text { Controls } \\
(n=94)\end{array}$ & $\begin{array}{l}\text { Welders } \\
(n=77)\end{array}$ & $\begin{array}{l}\text { Controls } \\
(n=94)\end{array}$ & $\begin{array}{l}\text { Welders } \\
(n=88)\end{array}$ & $\begin{array}{l}\text { Controls } \\
(n=69)\end{array}$ & & \\
\hline \multicolumn{9}{|c|}{ Continuous variables - median (5-95 percentile) } \\
\hline Age (years) & $43(23-59)$ & $44(23-56)$ & $49(29-65)$ & $50(29-63)$ & $43(25-60)$ & $42(25-58)$ & - & - \\
\hline Years welding & $10(1-25)$ & $0(0-11)$ & $15(5-31)$ & $0(0-11)$ & $7(1-27)$ & $0(0-5)$ & $<0.001$ & - \\
\hline $\begin{array}{l}\text { Respirable dust } \\
\qquad\left(\mathrm{mg} / \mathrm{m}^{3}\right)^{\mathrm{a}}\end{array}$ & $1.1(0.2-4.2)$ & - & $0.9(0.1-4.2)$ & - & $1.6(0.2-6.9)$ & - & 0.212 & \\
\hline $\begin{array}{l}\text { Respirable dust } \\
\text { adjusted }(\mathrm{mg} / \\
\left.\mathrm{m}^{3}\right)^{\mathrm{b}}\end{array}$ & $0.7(0.2-4.2)$ & - & $0.5(0.1-1.9)$ & - & $0.8(0.1-3.5)$ & - & 0.052 & - \\
\hline $\begin{array}{r}\text { Cumulative } \\
\text { exposure }^{c}\end{array}$ & $5.5(0.6-42.8)$ & - & $11.0(1.7-36.5)$ & - & $4.9(0.7-34.5)$ & - & $<0.001$ & - \\
\hline $\begin{array}{l}\text { Body-mass } \\
\text { index }\left(\mathrm{kg} / \mathrm{m}^{2}\right)\end{array}$ & $27.4(21.4-34.0)$ & $26.9(22.4-34.2)$ & $28.7(22.4-35.7)$ & $27.8(22.5-34.7)$ & $28.5(23.1-36.7)$ & $27.5(22.2-32.3)$ & $<0.001$ & $<0.001$ \\
\hline \multicolumn{9}{|c|}{ Categorical variables ${ }^{\mathrm{d}}-n(\%)^{\mathrm{e}}$} \\
\hline $\begin{array}{l}\text { Country of birth } \\
\text { (Sweden) }\end{array}$ & $55(71)$ & 88 (94) & $55(71)$ & 88 (94) & $61(69)$ & $62(91)$ & - & - \\
\hline $\begin{array}{l}\text { Education } \\
\text { (university or } \\
\text { higher) }\end{array}$ & $3(4)$ & $11(12)$ & $3(4)$ & $11(12)$ & $8(9)$ & $9(14)$ & $0.935^{\mathrm{j}}$ & $0.991^{\mathrm{j}}$ \\
\hline $\begin{array}{l}\text { Residence (large } \\
\text { and small } \\
\text { cities) }^{\mathrm{f}}\end{array}$ & $16(21)$ & $40(43)$ & $11(14)$ & $35(38)$ & $21(24)$ & $41(60)$ & 0.718 & 0.658 \\
\hline $\begin{array}{l}\text { Hobby exposure } \\
\text { to particles }\end{array}$ & $20(26)$ & $14(15)$ & $19(25)$ & $18(19)$ & $24(27)$ & $12(17)$ & 1 & 0.344 \\
\hline $\begin{array}{l}\text { Smoking history } \\
\text { (ever smoked) }\end{array}$ & $31(40)$ & $34(36)$ & $28(36)$ & $33(35)$ & $46(52)$ & $24(35)$ & 0.740 & 1 \\
\hline \multicolumn{9}{|l|}{$\begin{array}{l}\text { Smoking status } \\
\text { (currently) }\end{array}$} \\
\hline Non-smoker & $75(97)$ & $91(97)$ & $74(96)$ & $89(96)$ & $79(90)$ & $68(99)$ & 0.620 & 0.603 \\
\hline Party smoker & $2(3)$ & $3(3)$ & $1(1)$ & $2(2)$ & $8(9)$ & $1(1)$ & & \\
\hline Smoker & $0(0)$ & $0(0)$ & $2(3)$ & $2(2)$ & $1(1)$ & $0(0)$ & & \\
\hline Current snus use & $20(26)$ & $19(20)$ & $19(25)$ & $18(19)$ & $32(36)$ & $15(22)$ & 1 & 1 \\
\hline $\begin{array}{l}\text { Alcohol intake } \\
\text { ( } \geq 3 \text { times/ } \\
\text { week) }\end{array}$ & $2(3)$ & $2(2)$ & $2(3)$ & $3(3)$ & $1(1)$ & $4(6)$ & $0.956^{\mathrm{k}}$ & $0.832^{\mathrm{k}}$ \\
\hline $\begin{array}{l}\text { Vegetable intake } \\
\text { ( } \geq 5 \text { times/ } \\
\text { week })\end{array}$ & $47(61)$ & $61(66)$ & $42(55)$ & $68(73)$ & $51(58)$ & $45(65)$ & $0.653^{1}$ & $0.610^{1}$ \\
\hline $\begin{array}{l}\text { Physical activity } \\
\text { (moderate/ } \\
\text { high })^{\mathrm{h}}\end{array}$ & $29(38)$ & $40(43)$ & $36(47)$ & $46(49)$ & $41(47)$ & $29(42)$ & $0.254^{\mathrm{m}}$ & $0.680^{\mathrm{m}}$ \\
\hline Cancer history & $0(0)$ & $2(2)$ & $4(5)$ & $2(2)$ & $2(2)$ & $0(0)$ & 0.120 & 1 \\
\hline $\begin{array}{l}\text { Family cancer } \\
\text { history }\end{array}$ & $14(19)$ & $22(24)$ & $18(24)$ & $22(24)$ & $15(18)$ & $15(23)$ & 0.553 & 1 \\
\hline
\end{tabular}

${ }^{a}$ Measured by personal sampling or estimated; ${ }^{b}$ adjusted for personal respiratory protection equipment; ${ }^{c}$ cumulative exposure was calculated from adjusted respirable dust data and reported welding year experience; 'variables were categorized by 'yes' and 'no' unless otherwise stated; epercentage calculated relative to the total valid answers; ${ }^{\mathrm{f}}$ large and small cities as compared with towns and countryside; ${ }^{\mathrm{g}}$ exposure to welding fumes, dust, engine exhaust or engine diesel during leisure activities; ${ }^{\mathrm{h}}$ physical activity that involves sweating at least once a week and for at least $30 \mathrm{~min} ;{ }^{\mathrm{i}} p$-value for the differences between welders timepoint 1 and welders timepoint 2 calculated using paired samples Wilcoxon test for continuous variables and Fisher's exact test for categorical variables; ${ }^{j}$ statistical test based on five categories for education from secondary school to university studies; ${ }^{k}$ statistical test based on six categories for intake of alcohol from every day to never; ${ }^{1}$ statistical test based on eight categories from 3 per day or more to never; ${ }^{\mathrm{m}}$ statistical test based on four categories from sedentary to intensive physical activity; ${ }^{\mathrm{n}} p$ value for the differences between controls timepoint 1 and controls timepoint 2 calculated using paired samples Wilcoxon test for continuous variables and Fisher's exact test for categorical variables

of welding $(n=77)$, and cumulative exposure $(n=56)$ in welders only (Table 3 ). Since years of welding and cumulative exposure were correlated with age $\left(r_{S}=0.53\right.$,
$\mathrm{r}_{\mathrm{S}}=0.30$, respectively), we included models both with and without age as a co-variate, as well as models in which age was the independent variable (Table 3). For SDC1, 


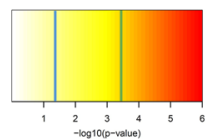

Timepoint 1

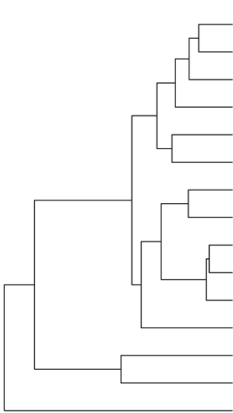

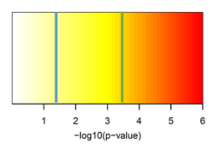

Timepoint 2
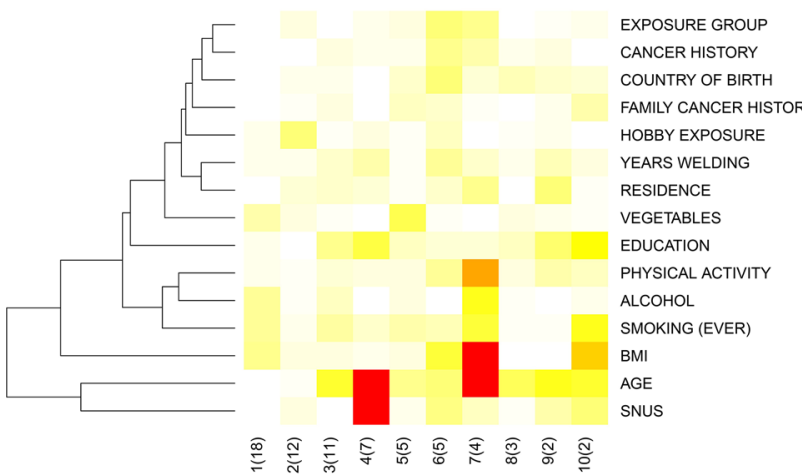

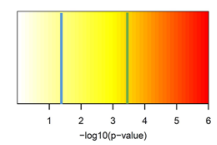

Cross-sectional group

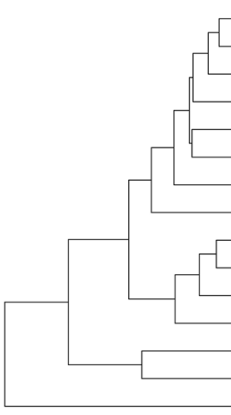

CANCER HISTORY

SMOKING (EVER)

EDUCATION

VEGETABLES

HOBBY EXPOSURE

ALCOHOL

FAMILY CANCER HISTORY

COUNTRY OF BIRTH

EXPOSURE GROUP

PHYSICAL ACTIVITY

RESIDENCE

YEARS WELDING

AGE

BNUS

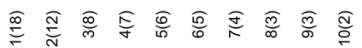

Fig. 2 Heatmaps of the principal components (PC) that explain the variation in the study groups. Numbers on the horizontal axis of the heatmaps indicate number of the principal component and in brackets is the percentage of the explained variation in the protein dataset. Heatmaps were constructed using input data from linear regression of association between the principal components of the data and the biological annotations. The influence of the biological annotations on the overall variation is plotted in a heatmap based on the $p$ value of the association. Input data were the normalized protein expression values (on a $\log 2$-scale). Lines in the key indicate $p$ value thresholds (blueunadjusted; green-Bonferroni-adjusted for multiple comparisons). 'group' refers to welders and controls; 'residence' refers to current

age alone was associated with the proteins levels (in models adjusted for BMI and snus) and it is, therefore, difficult to disentangle the contribution of age from the contribution of welding years or cumulative exposure. However, for FOLR1, the protein levels were associated with years of welding irrespective of age. Similar associations between age and the respective circulating proteins were identified in controls. residence in large and small cities as compared with towns and countryside; 'hobby exposure' exposure to welding fumes, dust, engine exhaust, or engine diesel during leisure activities; 'country of birth' is categorized as Sweden or outside Sweden; 'education' is assigned to five categories for education from secondary school to university studies; 'vegetables' frequency of intake of vegetables and is assigned to eight categories from 3 per day or more to never; "physical activity' is based on four categories from sedentary to intensive physical activity; 'ever smoking' stands for current or previous smoking and is categorized as 'yes' and 'no'; 'alcohol' stands for frequency of alcohol intake and is stratified in six categories from every day to never (color figure online)

\section{Discussion}

The current study identified 14 putative cancer-related proteins that were differentially expressed in a longitudinal cohort of welders working with mild steel. Associations with three of these proteins (SDC1, FOLR1, and SPARC) were similarly altered in an additional cross-sectional set 
Table 2 Differentially expressed proteins in serum between welders and controls in the main study group (linear mixed models) and corresponding data for the cross-sectional group (linear models)

\begin{tabular}{|c|c|c|c|c|c|c|}
\hline \multirow[t]{2}{*}{ Protein } & \multicolumn{3}{|c|}{ Linear mixed models $(n=342)$} & \multicolumn{3}{|c|}{$\begin{array}{l}\text { Linear models (cross-sectional group) } \\
(n=157)\end{array}$} \\
\hline & $R_{\mathrm{m}}^{2}(\%)^{\mathrm{a}}$ & $\beta(\mathrm{SE})^{\mathrm{b}}$ & $p^{\mathrm{c}}$ & $R^{2}(\%)^{\mathrm{d}}$ & $\beta(\mathrm{SE})^{\mathrm{e}}$ & $p^{\mathrm{f}}$ \\
\hline $\mathrm{SDC} 1 *$ & 20 & $-0.288(0.049)$ & $<0.001^{\#}$ & 14 & $-0.311(0.066)$ & $<0.001$ \\
\hline FOLR1* & 7 & $-0.127(0.038)$ & 0.001 & 8 & $-0.157(0.049)$ & 0.002 \\
\hline SPARC* & 4 & $-0.044(0.021)$ & 0.034 & 5 & $-0.051(0.025)$ & 0.046 \\
\hline KLK8 & 19 & $0.103(0.05)$ & 0.040 & 2 & $0.130(0.066)$ & 0.051 \\
\hline CRNN & 35 & $-0.385(0.136)$ & 0.004 & 36 & $0.037(0.167)$ & 0.827 \\
\hline CEACAM1* & 4 & $-0.047(0.017)$ & 0.005 & 0 & $-0.027(0.025)$ & 0.271 \\
\hline ANXA1* & 3 & $0.190(0.07)$ & 0.007 & 2 & $0.107(0.101)$ & 0.292 \\
\hline PODXL* & 9 & $-0.062(0.026)$ & 0.015 & 2 & $-0.030(0.028)$ & 0.293 \\
\hline EPHA2* & 3 & $-0.067(0.028)$ & 0.017 & 3 & $-0.065(0.037)$ & 0.081 \\
\hline DLL1* & 7 & $-0.088(0.037)$ & 0.019 & 3 & $-0.06(0.047)$ & 0.203 \\
\hline SEZ6L & 15 & $-0.069(0.031)$ & 0.024 & 8 & $-0.031(0.036)$ & 0.391 \\
\hline FASLG* & 28 & $-0.128(0.057)$ & 0.024 & 22 & $0.062(0.058)$ & 0.289 \\
\hline LYPD3 & 20 & $-0.089(0.04)$ & 0.025 & 6 & $-0.022(0.052)$ & 0.668 \\
\hline CYR61* & 17 & $-0.104(0.048)$ & 0.029 & 18 & $-0.018(0.051)$ & 0.725 \\
\hline
\end{tabular}

$S E$ standard error; ${ }^{a}$ Variance explained by fixed factors (group, age, body-mass index, use of snus); ${ }^{b}$ regression coefficient from linear mixed models interpreted as standard deviation difference in protein levels compared to controls, adjusted for age, body-mass index, and use of snus variables as fixed factors, and participant as random factors; ${ }^{c} p$ value from test of contribution of group inclusion (welders and controls) to protein variance using an analysis of variance approach with Satterthwaite approximation for degrees of freedom (Bonferroni-adjusted threshold for the $p$ value: $0.05 / 91=5.5 \times 10^{-4}$ ); ${ }^{\mathrm{d}}$ variance in protein levels explained by the linear model; ${ }^{e}$ regression coefficient from multivariable-adjusted linear models interpreted as standard deviation difference in protein levels compared to controls adjusted for age, body-mass index, and use of snus; ${ }^{\mathrm{f}} p$ value from the linear model to test the difference between welders and controls; ${ }^{*}$ significant after adjustment for multiple testing (Bonferroni); SDC1, FOLR1, and SPARC are the only three proteins significant in both analyses; *gene expressed in lung tissue (according to the Human Protein Atlas, www.proteinatlas.org) of welders and controls. Importantly, these associations are observed at levels of moderate exposure to welding fumes and clearly below the current Swedish occupational exposure limit $\left(2.5 \mathrm{mg} / \mathrm{m}^{3}\right)$. Further studies are needed to conclude if these protein alterations are true markers of later disease.

The top 20 differentially expressed proteins were found to be related to functions such as cellular movement (cell migration and invasion) which is a potentially novel mechanism of toxicity for welding fumes. There was no association with proteins involved in inflammation or immune response, e.g., interleukin 6 or the Toll-like receptor 3. However, annexin A1, an anti-inflammatory glucocorticoid-regulated protein (Sugimoto et al. 2016), was upregulated in welders compared with controls. Overall, studies evaluating associations between welding fumes and inflammation show inconsistent results, and the contribution of inflammation to welding-induced lung cancer is still unclear. Changes in inflammatory status have been observed following acute exposure to welding (Kauppi et al. 2015; Kim et al. 2005; Shen et al. 2018; Wang et al. 2005), but there are only a few studies addressing inflammation after long-term exposure to welding fumes and with no clear associations $(\mathrm{Li}$ et al. 2015a, b). Indeed, the role of inflammation in welding-related cancer needs to be further evaluated in further studies. In addition, several studies in welders found that smoking can profoundly affect the status of inflammation (Kim et al. 2005; Wang et al. 2005), and therefore, it might be difficult to disentangle the contribution of smoking from the effect of exposure to welding fumes. In the current study, we can exclude the potential contribution of smoking to the observed associations, since, with very few exceptions, study participants were non-smokers. The few smokers were excluded in a sensitivity analysis, without altering the results.

SDC1, the most significant protein differentially expressed in welders, was downregulated in welders versus controls, and was the only protein that passed the significance threshold after adjusting for multiple testing. SDC1 is expressed in different tissues of epithelial origin, including the lung (proteinatlas.org). It is a cell surface bound heparan sulfate proteoglycan involved in numerous cell processes such as growth, adhesion, and migration (Szatmari et al. 2015). SDC1 is constitutively shed from the surface of the cells, a process that is mediated by proteases (e.g., heparanase) and is accelerated in 

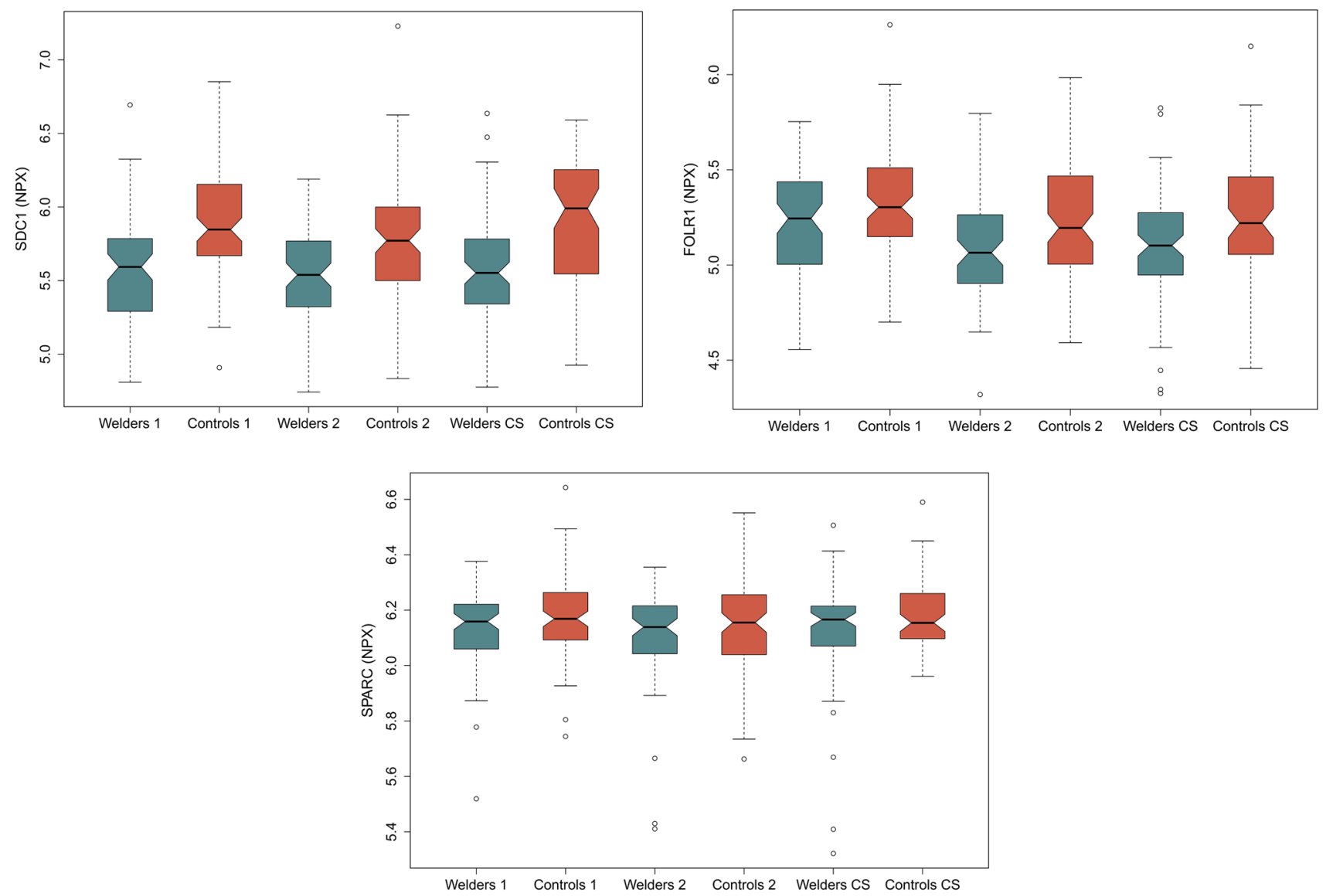

Fig. 3 Box plots of normalized protein expression (NPX) values for SDC1, FOLR1, and SPARC in the six study groups. The $p$ values $(t$ test) for the comparisons between the welders and controls for the dif-

connection to tumors (Ramani et al. 2013). SDC1 can act both a tumor suppressor and a tumor promoter, and this dual effect depends on the tissue of origin and form (cell surface bound or soluble) (Garusi et al. 2012). High levels of SDC1 in serum are associated with a poor prognosis in, for example, multiple myeloma (Seidel et al. 2000) and lung cancer (Joensuu et al. 2002). The latter study reported, however, no association between SDC1 tissue expression and serum levels, albeit on a small sample size $(n=45)$ (Joensuu et al. 2002). On the other hand, SDC1 in serum was significantly lower in individuals with malignant pleural mesothelioma as compared with unexposed controls or individuals exposed to asbestos but without mesothelioma (Demir et al. 2016).

FOLR1 was another protein differentially expressed in welders (downregulated) and, in addition, negatively associated with years of welding. FOLR is expressed in different epithelial tissues, with a high expression in the lung (proteinatlas.org). It is a cell membrane receptor involved in folate transport and predominantly present on the surface of epithelial cells (Elnakat and Ratnam 2004). It can also be shed ferent timepoints were significant $(<0.05)$ for all proteins apart for SPARC comparison welder timepoint 2 versus control timepoint 2 and welders cross-sectional versus control cross-sectional

from the surface of the cells and reach the blood stream in a soluble form (Kelemen et al. 2014). Increased serum levels of FOLR1 have been associated with ovarian cancer and there was a good correlation between expression in tumor tissue and soluble FOLR1 (Kurosaki et al. 2016). Increased expression of FOLR1 at tissue level was associated with malignant pleural mesothelioma and lung adenocarcinoma (Bueno et al. 2001; O'Shannessy et al. 2012).

SPARC was downregulated in welders compared with the controls, but without any association with exposure to welding measured as welding years, respirable dust, or cumulative exposure. SPARC is predominantly found as a secreted glycoprotein, but it is also present in the cell membrane and intracellularly (Wong and Sukkar 2017). Extracellular SPARC mediates cell-extracellular matrix interactions as well as angiogenesis and in the lung SPARC is involved in development of fibrosis and progression of lung cancer (Wong and Sukkar 2017). SPARC expression in lung squamous cell carcinoma tissue was reported to be higher compared to non-cancerous tissue and was significantly correlated with poor outcome in lung squamous cell carcinoma 


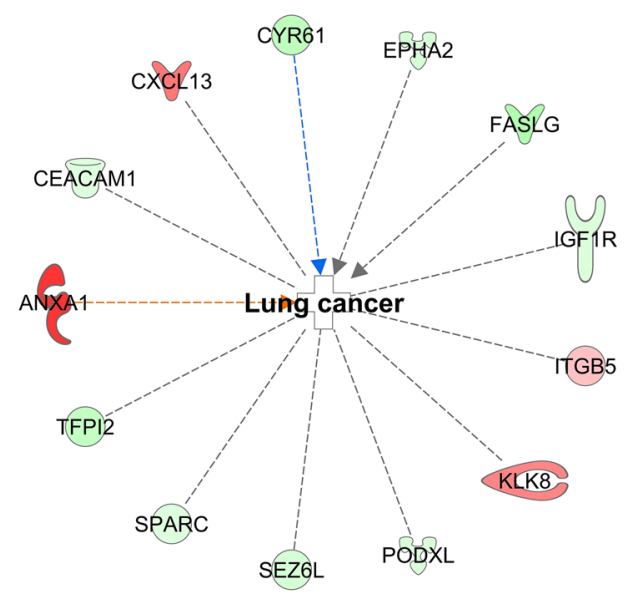

Fig. 4 Networks of the most significant diseases and functions defined by the differentially expressed proteins between welders and the controls in the linear mixed models. Analysis was performed using Ingenuity Pathway Analysis for the top 20 proteins $(p<0.1)$.
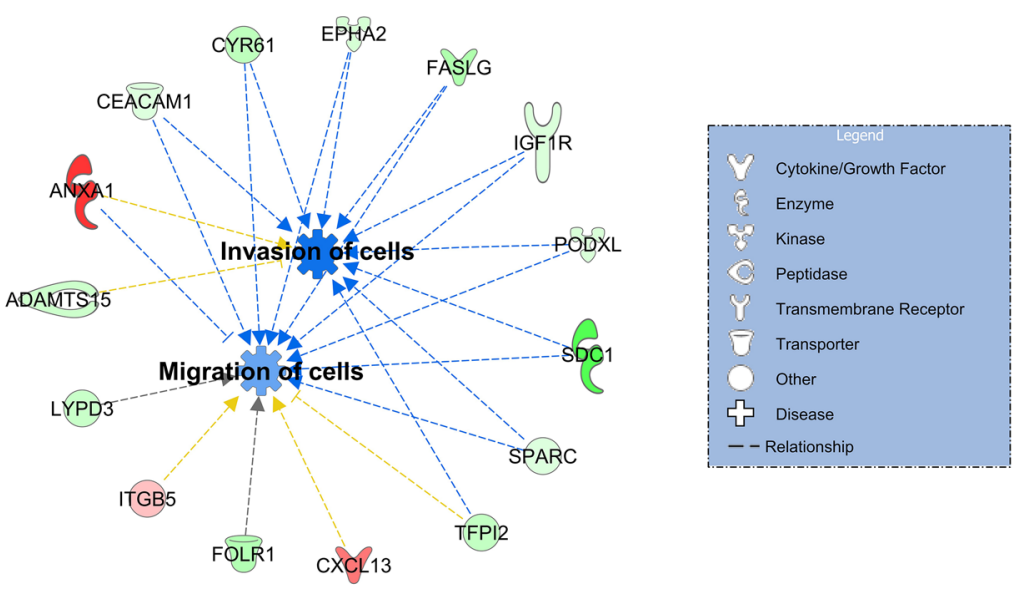

Proteins represented in red are upregulated, whereas proteins represented in green are downregulated in welders versus controls (color figure online)
Table 3 Associations of the differentially expressed proteins in welders and controls (SDC1, FOLR1, SPARC) with exposure to welding expressed as respirable dust (adjusted for personal respiratory protec- tive equipment), years welding and cumulative exposure in welders only (linear mixed models)

\begin{tabular}{|c|c|c|c|c|c|c|c|c|c|}
\hline & \multicolumn{3}{|l|}{ SDC1 } & \multicolumn{3}{|l|}{ FOLR1 } & \multicolumn{3}{|l|}{ SPARC } \\
\hline & $R_{\mathrm{m}}^{2}(\%)^{\mathrm{a}}$ & $\beta(\mathrm{SE})^{\mathrm{b}}$ & $p^{\mathrm{c}}$ & $R_{\mathrm{m}}^{2}(\%)^{\mathrm{a}}$ & $\beta(\mathrm{SE})^{\mathrm{b}}$ & $p^{\mathrm{c}}$ & $\mathrm{R}_{\mathrm{m}}^{2}(\%)^{\mathrm{a}}$ & $\beta(\mathrm{SE})^{\mathrm{b}}$ & $p^{\mathrm{c}}$ \\
\hline \multicolumn{10}{|c|}{ Respirable dust $(n=112)$} \\
\hline Age, BMI, and snus & 15 & $0.022(0.033)$ & 0.501 & 10 & $-0.020(0.030)$ & 0.504 & 3 & $0.011(0.016)$ & 0.487 \\
\hline \multicolumn{10}{|l|}{ Years welding $(n=154)$} \\
\hline BMI and snus & 6 & $-0.008(0.004)$ & 0.044 & 9 & $-0.007(0.003)$ & 0.024 & 2 & $0.000(0.002)$ & 0.912 \\
\hline Age, BMI and snus & 8 & $-0.002(0.005)$ & 0.618 & 10 & $-0.009(0.004)$ & 0.017 & 3 & $0.001(0.002)$ & 0.513 \\
\hline \multicolumn{10}{|c|}{ Cumulative exposure $(n=112)$} \\
\hline BMI and snus & 8 & $-0.009(0.003)$ & 0.011 & 12 & $-0.005(0.003)$ & 0.069 & 3 & $0.002(0.001)$ & 0.290 \\
\hline Age, BMI, and snus & 16 & $-0.004(0.004)$ & 0.242 & 12 & $-0.004(0.003)$ & 0.126 & 6 & $0.003(0.002)$ & 0.102 \\
\hline \multicolumn{10}{|l|}{ Age $(n=154)$} \\
\hline BMI and snus & 8 & $-0.008(0.003)$ & 0.007 & 6 & $-0.001(0.003)$ & 0.593 & 2 & $-0.001(0.001)$ & 0.504 \\
\hline
\end{tabular}

$S E$ standard error; ${ }^{a}$ Variance explained by fixed factors (respirable dust/years welding/cumulative exposure, age, body-mass index, and use of snus); ${ }^{b}$ regression coefficient from linear mixed models interpreted as standard deviation difference in protein levels per respirable dust unit increase/numbers of years welding/cumulative exposure unit increase, adjusted for age, body-mass index, and use of snus variables as fixed factors, and participant as random factors; ${ }^{c} p$ value from test of contribution of respirable dust/years welding/cumulative exposure to protein variance using an analysis of variance approach with Satterthwaite approximation for degrees of freedom

patients (Xu et al. 2019). SPARC levels were found to be higher in plasmas of lung cancer patients compared with healthy heavy-smoking controls and SPARC was suggested to be a diagnostic tool to discriminate between the two groups (Andriani et al. 2018).

The directions (lower in welders versus controls, or in relation to years welding) found in our study for SDC1, FOLR1, and SPARC were in several cases opposite to the direction found for protein levels in serum of cancer patients versus controls, or in relation to poor cancer prognosis. It is, therefore, unclear at this point to which extent the associations that we found represent toxic or adaptive responses following exposure to welding fumes, or if these putative cancer biomarkers are predictive of future cancer risk. In addition, it should be stressed that the study participants are healthy, even though they are exposed to potentially carcinogenic welding fumes and the associations might be different for healthy individuals compared to cancer patients. Moreover, the interplay between tissue and serum levels of proteins is highly dynamic and complex, and renders it difficult to compare studies that evaluate tissue levels with studies that evaluate serum levels of proteins.

It is yet unclear why FOLR1 seems to decrease from timepoint 1 to timepoint 2 both in welders and in controls. 
One possible explanation could be that serum levels of these proteins decrease with age. We could only find one reference in the literature where FOLR1 was reported to be associated with age; however, the study indicated a positive association that was only valid for women (Kelemen et al. 2014). In addition, we investigated whether the changes could be related with storage time of the samples, but we found no indication of such associations. It should be noted that the sampling was performed using the same protocol, by the same nurse and using same collection tubes. Since the sampling was performed approximately 6 years apart, we cannot exclude a batch effect. If that was the case, we do not expect that such a batch effect would have an impact on the observed associations with serum levels of cancer-related proteins, since both welders and controls were sampled at both timepoints.

Downstream analysis, i.e., disease and function analysis, performed on the top 20 proteins differentially expressed in welders indicated lung cancer as top disease and cell movement as one of the top functions. While the analysis predicted an inhibition of cell movement based on the input data, the direction of this prediction bears a great deal of uncertainty. This is mainly due to the background data at the basis of this prediction, as there are few data for serum samples. Furthermore, the correlations between the tissues and serum levels of the respective cancer-related proteins are yet not well established. We emphasize that these enrichments are merely prediction-based and additional validation should be performed in mechanistic studies of welding fumes.

Using a similar approach as in our study, cornulin [CRNN, member of the 'fused gene' family (Contzler et al. 2005)], levels in plasma were previously reported to be associated with consumption of snus (Sundkvist et al. 2018). CRNN was also differentially expressed in welders in our longitudinal analysis, albeit this was not replicated in the cross-sectional analysis. Interestingly, the variance explained by the model for CRNN is relatively high (34\%) and this is likely related to the presence of snus in the model. Moreover, snus was one of the factors that was indicated in the PCA heatmap as being significant in explaining the variation in the data set, and this was conditioned by the presence of CRNN. The mechanisms behind the association between CRNN and snus have yet to be clarified.

One of the strengths of this study is the combination of a longitudinal approach with a cross-sectional approach. The longitudinal analysis takes into account intra-individual variations and time-dependent changes and provides the basis for a hypothesis generation, while the cross-sectional approach increases the study validity by allowing for replication of the longitudinal findings in an additional sample set. Moreover, we had access to extensive data on the background and lifestyle of the study participants (e.g., diet, physical activity, alcohol intake, cancer history, and hobbies) that could confound the statistical analyses. The number of smokers was marginal, as smoking was one of the exclusion criteria at recruitment. This is important, since smoking is a frequent confounder when cancer-related outcomes are evaluated. We also had information on hobby exposure to welding or other hobbies that incur increased exposure to carcinogenic particles. In additional to the group analysis, welders versus controls, we also modeled the relationships with different types of exposure measurements in welders only. This was facilitated by having information on the number of years welding and welding frequency, as well as by measuring the amount of respirable dust, individual welders are exposed to. One of the limitations of the latter approach is the lower power due to a reduced number of welders that had their respirable dust assessed at two different occasions $(n=56)$.

\section{Conclusions}

In this study, we identified associations between moderate exposure to welding fumes and cancer-related proteins in blood. It is yet unclear to which extent these changes are adaptive or predictive of cancer risk, and further studies are warranted.

\section{Methods}

\section{Study design}

This investigation is based on a cohort of welders established in 2010 in the south of Sweden (Södra sjukvårdsregionen) (Li et al. 2015a). At baseline, we recruited 101 welders working in small- and medium-size welding companies and 127 age-matched controls working in small companies, but without occupational exposure to particles, including from welding fumes. The inclusion criteria were that they should be non-smokers since at least 6 months and male. However, at follow-up, we identified based on the questionnaire data a few individuals that actually were current smokers. The follow-up after 6 years was performed in 2016-2017 with a dropout of 23\% $(n=23)$ among welders and $24 \%(n=31)$ among controls; the main reason for dropout was retirement and closure of one welding company. At follow-up, we recruited new participants (67 welders and 38 controls) with the same inclusion criteria. The follow-up survey included examination of 145 welders ( 78 welders re-examined) and 134 controls (96 controls re-examined). In this study, the participants were divided into two groups: one group with repeated measurements, i.e., cohort group (2010/2011cohort timepoint 1 and 2016/2017-cohort timepoint 2), and one group that had measurements performed at either baseline or follow-up, i.e., cross-sectional group (90 welders 
and 69 controls). Out of the latter group, three welders and two controls were excluded from the analysis due to incomplete questionnaire and/or low quality for the protein measurements. In addition, two controls and one welder from the cohort were re-attributed to the cross-sectional group due to incomplete questionnaire and/or low quality for the protein measurements for one of the cycles. This amounts to a total of 142 individuals in the cohort group (77 welders and 94 controls) and 157 individuals in the cross-sectional group (88 welders and 69 controls). A flow diagram of the study design is included in Fig. 1.

Study participants were asked to fill out a questionnaire regarding country of birth, education (five categories: primary school, high-school, professional school, university $<3$ years, and university $>3$ years), medical history, personal/family history of cancer, diet (frequency of intake of fruit vegetables, fish), physical activity (from sedentary to intense exercise), current as well as previous smoking history, use of snus (Swedish moist tobacco, yes/no), alcohol consumption (six categories from never to every day), current residence (four categories; large city, small city, town, countryside), and exposure to particles/smoke (e.g. welding fumes, dust, engine exhaust, diesel engine) during leisure activities.

Blood samples were collected in BD vacutainers for serum, allowed to clot at room temperature for $10 \mathrm{~min}$, and then centrifuged at $2400 \mathrm{rcf}$ for $10 \mathrm{~min}$. Upon separation, serum samples were aliquoted and kept on dry ice for transportation to the laboratory in the Division of Occupational and Environmental Medicine at Lund University, and then stored at $-80^{\circ} \mathrm{C}$ until analysis.

\section{Exposure assessment}

For occupational history, we used, both for welders and controls, a structured questionnaire inquiring about the present and past workplaces, type, and duration of job and explicitly whether workers were exposed to welding or diesel fumes at past or present workplaces.

We asked the welders, in addition to the above-mentioned questions, about the type of welding, total hours welding during the usual working week, place of welding, area-level or point source exhaust use, as well as use of personal respiratory, noise, and eye protection devices while welding.

\section{Personal respirable dust measurement}

Personal sampling of respirable dust was performed for welders and area-level respirable dust monitoring was mainly performed for controls. For the personal sampling, a filter cassette was placed within the personal breathing zone of welders. Respirable dust was collected on $37 \mathrm{~mm}$ mixed cellulose ester filters with $0.8 \mu \mathrm{m}$ pore size (pre-weighed), fitted in conductive cassettes attached to personal sampling cyclones (BGI4L, BGI, Mesa Labs, USA; cut-off $4 \mu \mathrm{m}$ ). The airflow through the sampler was set at $2.2 \mathrm{~L} / \mathrm{min}$ and regularly checked before, during, and after sampling with primary calibrators (TSI Model 4100 Series, TSI Incorporated, USA). Personal sampling was coordinated with shift working hours; an average time of sampling was $6 \mathrm{~h}$ and $33 \mathrm{~min}(2 \mathrm{~h}$ and $22 \mathrm{~min}$ to $8 \mathrm{~h}$ and $33 \mathrm{~min}$ ) for timepoint 1 and $6 \mathrm{~h}$ and $33 \mathrm{~min}(3 \mathrm{~h}$ and $5 \mathrm{~min}$ to $8 \mathrm{~h}$ and $29 \mathrm{~min}$ ) for timepoint 2. Measured concentration was corrected for if respiratory protection was used, a factor of three was used to reduce measured concentration (outside respiratory protection) to actual exposure (Hedmer et al. 2014; Li et al. 2015a). At timepoint 2, one welder used a half-mask and then a correction factor of two was used, and four welders used newer versions of powered air purifying respirators with double visors and then a factor 50 was used. The filter samples were analyzed gravimetrically according to a validated method for determination of respirable dust. The limit of detection was set to $0.05 \mathrm{mg} / \mathrm{sample}$. For those welders missing measured exposure data, the exposure was assessed individually using exposure data from welders working at the same location, engaged in similar tasks, and in the same company. Exposure data previously collected at the welding companies were also used in the exposure assessments (Hedmer et al. 2014; Li et al. 2015b). In total, there were 101 welders that had respirable dust data at timepoint 1 (measured respirable dust $n=53$; estimated respirable dust $n=48)$ and 123 that had respirable dust data at timepoint 2 (measured respirable dust $n=103$; estimated respirable dust $n=20$ ). There were 56 welders that had respirable dust data at both timepoints (timepoint 1: measured $n=28$, estimated $n=28$; timepoint 2: measured $n=46$, and estimated $n=10$ ).

In control companies at timepoint 1 , full-shift personal breathing zone samples of respirable dust were collected in two companies for 19 controls. In four companies, area-level air pollution monitoring of respirable dust was performed using a direct reading monitor, SidePak Model AM510 (TSI Incorporated) with a Dorr-Oliver cyclone ( $\mathrm{Li}$ et al. 2015a). In control companies at timepoint 2 , area-level air pollution monitoring of respirable dust fractions was performed using DustTrak DRX monitor (TSI Incorporated). The monitors were placed at breathing zone height in the area where workers spent the most time during the work shift. In companies where workers spent time at two different workstations, measurement was performed in both areas, but divided before and after the lunch break. All statistical analyses and calculation of cumulative dose were performed using respirable dust values that were adjusted for personal respiratory protection, as they better reflect the actual exposure.

\section{Cumulative dose}

For timepoint 1, the cumulative dose was estimated by multiplying respirable dust (adjusted for PPE) and reported years 
of welding experience. For timepoint 2, similar calculation was performed, and the estimate from timepoint 1 was added to calculate cumulative dose:

Cumulative dose timepoint_1 $_{\text {tirable dust }}=$ Respimepoint_1 $\times$ Years welding ${ }_{\text {timepoint_1 }}$

Cumulative dose timepoint_2 $_{\text {timepoint_1 }}=$ Cumulative dose $+\left[\right.$ Respirable dust timepoint_2 $_{2} \times$ (Years welding timepoint_2 $_{2}$. -Years welding timepoint_1 $_{1}$ ]

\section{Measurement of proteins in serum}

Serum samples were analyzed for 92 unique proteins using the Proseek Multiplex Oncology II kit coupled with the Fluidigm BioMark HD real-time PCR platform (Olink Proteomics, Uppsala, Sweden). The kit contains proteins that are curated to ontologies relevant for cancer development: angiogenesis $(n=20)$, apoptotic process $(n=34)$, cell adhesion $(n=35)$, cell differentiation $(n=42)$, cell motility $(n=30)$, cell proliferation $(n=43)$, cellular metabolic process $(n=47)$, cellular response to stress $(n=23)$, chemotaxis $(n=14)$, extracellular matrix organization ( $n=9)$, immune response $(n=27)$, MAPK cascade $(n=25)$, proteolysis $(n=19)$, response to hypoxia $(n=3)$, and other gene ontology terms $(n=9)$. The analysis is based on the proximity extension assay (PEA); processing, quality control as well as normalization were previously described (Assarsson et al. 2014). Protein levels are reported as normalized protein eXpression (NPX) values on a log2-scale. The cut-off for intraor inter-assay $\mathrm{CVs}$ were $<20 \%$. All proteins had an intra-assay coefficient of variation (CV) lower than $13 \%$. The majority of the proteins had an inter-assay CV lower than $16 \%$. XPNPEP2 had an inter-assay CV of $33 \%$ and was excluded from the analysis. CRNN had an inter-assay CV of $22 \%$, but was kept in the analysis due to the relevance of CRNN for the use of snus (Sundkvist et al. 2018). All the downstream analyses were performed on 91 proteins.

\section{Statistical analyses}

\section{Evaluation of differences between the study groups}

Characteristics are presented as median and 5-95 percentile for the continuous variables and percentage for categorical variables. Differences between groups were evaluated with the Kruskal-Wallis rank sum test (followed by Dunn's post hoc test) (when comparing three groups) and paired samples Wilcoxon test (when comparing two groups) for continuous variables, as well as Fisher's exact test for categorical variables.

\section{Data exploration using principal component analysis}

PCA heatmaps were constructed using the prince.plot function in the swamp package in $R$. The function generates principal components that explain part of the variation in the protein data set and then tests each variable against these components to evaluate possible associations. Heatmaps depict $p$ values ( $-\log 10$-transformed) of these associations. Hierarchical clustering of the variables was generated using the hclust function.

\section{Evaluation of differentially expressed proteins}

Longitudinal analysis employed linear mixed models to evaluate associations between groups (welder and control) with cancer-related proteins were fitted using the lmer function in the lme4 package in $R$. The mixed models included participants as random factors (random intercepts) and age, body-mass index, use of snus as well as group as fixed factors. Variance explained by fixed factors $\left(R_{\mathrm{m}}^{2}\right)$ and random factor $\left(R_{\mathrm{c}}^{2}\right)$ was calculated using RsqGLM function from the $R$ package MuMin. Models were assessed by plotting Pearson standardized residuals. Sensitivity analyses were performed (1) only on the non-smokers ( $n=74$ welders, $n=89$ controls) and (2) by excluding welders with respirable dust levels $>2.5 \mathrm{mg} / \mathrm{m}^{3}(n=10)$.

For the cross-sectional analysis of the cross-sectional group, we used multivariable-adjusted linear models (adjusted for age, body-mass index, and use of snus) to evaluate the associations between groups (welder amd controls) with cancer-related proteins.

Similar analyses using linear mixed models were performed in welders only by replacing the group variable with respirable dust (in $\mathrm{mg} / \mathrm{m}^{3}$ ), years of welding (in years), or cumulative exposure. The mixed models included participants as random factors (random intercepts) and age, bodymass index, use of snus as well as respirable dust, years of welding, or cumulative exposure as fixed factors. The longitudinal analysis was performed on 54 welders (sampled twice) for the association with respirable dust and cumulative exposure and on 77 welders (sampled twice) for the association with years of welding.

\section{Bioinformatics analysis of the differentially expressed proteins}

Disease and function analysis was performed using Ingenuity Pathway Analysis (license obtained from Ingenuity Systems, Redwood City, CA, USA) on the top 20 differentially expressed proteins between welders and controls 
from the linear mixed model analysis. All proteins had a $p$ value $<0.1$. We changed the significance threshold from 0.05 to 0.1 in the disease and function analysis to increase the number of proteins and the statistical power for the downstream bioinformatics analysis.

All analyses ware performed using R v.3.3.2 (R Foundation for Statistical Computing, Vienna, Austria) unless otherwise stated.

Acknowledgements Open access funding provided by Karolinska Institute. This work was supported by the Swedish Research Council FORTE, Karolinska Institutet, and the Clinic of Occupational and Environmental Medicine, Lund University Hospital. We thank all the workers who participated in this study. We would also like to thank Dr. Huiqi Li for the assistance during sampling of timepoint 1, Dr. Ayman Alhamdow for help with the preparation of the serum samples as well as Dr. Jakob Riddar, Ulf Bergendorf, Jan-Eric Karlsson, Åsa Amilon, and Dr. Annette Krais for assisting during the sampling of respirable dust.

\section{Compliance with ethical standards}

Conflict of interest The authors declare that they have no competing interests.

Availability of data and materials The data sets used and/or analyzed during the current study are available from the corresponding author on reasonable request.

Ethical approval All procedures performed in studies involving human participants were in accordance with the ethical standards of the institutional and/or national research committee (Regional Ethical Committee of Lund University, Sweden) and with the 1964 Helsinki Declaration and its later amendments or comparable ethical standards.

Informed consent Informed consent was obtained from all individual participants included in the study.

Open Access This article is distributed under the terms of the Creative Commons Attribution 4.0 International License (http://creativeco mmons.org/licenses/by/4.0/), which permits unrestricted use, distribution, and reproduction in any medium, provided you give appropriate credit to the original author(s) and the source, provide a link to the Creative Commons license, and indicate if changes were made.

\section{References}

Andriani F et al (2018) Diagnostic role of circulating extracellular matrix-related proteins in non-small cell lung cancer. BMC Cancer 18:899

Assarsson E et al (2014) Homogenous 96-Plex PEA immunoassay exhibiting high sensitivity, specificity, and excellent scalability. PLoS One 9:e95192

Bueno R, Appasani K, Mercer H, Lester S, Sugarbaker D (2001) The alpha folate receptor is highly activated in malignant pleural mesothelioma. J Thorac Cardiovas Surg 121:225-233

Contzler R, Favre B, Huber M, Hohl D (2005) Cornulin, a new member of the "fused gene" family, is expressed during epidermal differentiation. J Invest Dermatol 124:990-997
Demir M et al (2016) Evaluation of new biomarkers in the prediction of malignant mesothelioma in subjects with environmental asbestos exposure. Lung 194:409-417

Elnakat H, Ratnam M (2004) Distribution, functionality and gene regulation of folate receptor isoforms: implications in targeted therapy. Adv Drug Deliv Rev 56:1067-1084

Garusi E, Rossi S, Perris R (2012) Antithetic roles of proteoglycans in cancer. Cell Mol Life Sci 69:553-579

Grigg J, Miyashita L, Suri R (2017) Pneumococcal infection of respiratory cells exposed to welding fumes; Role of oxidative stress and HIF-1 alpha. PLoS One 12:e0173569

Guha N et al (2017) Carcinogenicity of welding, molybdenum trioxide, and indium tin oxide. Lancet Oncol 18:581-582

Han SG, Kim Y, Kashon ML, Pack DL, Castranova V, Vallyathan V (2005) Correlates of oxidative stress and free-radical activity in serum from asymptomatic shipyard welders. Am J Respir Crit Care Med 172:1541-1548

Hedmer M, Karlsson JE, Andersson U, Jacobsson H, Nielsen J, Tinnerberg H (2014) Exposure to respirable dust and manganese and prevalence of airways symptoms, among Swedish mild steel welders in the manufacturing industry. Int Arch Occup Environ Health 87:623-634

Hoffmeyer F et al (2012a) Impact of different welding techniques on biological effect markers in exhaled breath condensate of 58 mild steel welders. J Toxicol Environ Health Part A 75:525-532

Hoffmeyer F et al (2012b) Relation between biomarkers in exhaled breath condensate and internal exposure to metals from gas metal arc welding. J Breath Res 6:027105

Hossain MB, Li H, Hedmer M, Tinnerberg H, Albin M, Broberg K (2015) Exposure to welding fumes is associated with hypomethylation of the F2RL3 gene: a cardiovascular disease marker. Occup Environ Med 72:845-851

IARC (2019) Welding, Molybdenum Trioxide, and Indium Tin Oxide. IARC monographs on the evaluation of carcinogenic risks to humans, vol 118. International Agency for Research on Cancer, Lyon

Joensuu H, Anttonen A, Eriksson M, Makitaro R, Alfthan H, Kinnula V, Leppa S (2002) Soluble syndecan-1 and serum basic fibroblast growth factor are new prognostic factors in lung cancer. Cancer Res 62:5210-5217

Kauppi P et al (2015) Systemic inflammatory responses following welding inhalation challenge test. Toxicol Rep 2:357-364

Kelemen LE, Brenton JD, Parkinson C, Whitaker HC, Piskorz AM, Csizmadi I, Robson PJ (2014) Conditions associated with circulating tumor-associated folate receptor 1 protein in healthy men and women. PLoS One 9:e96542

Kim JY, Chen JC, Boyce PD, Christiani DC (2005) Exposure to welding fumes is associated with acute systemic inflammatory responses. Occup Environ Med 62:157-163

Kurosaki A et al (2016) Serum folate receptor alpha as a biomarker for ovarian cancer: implications for diagnosis, prognosis and predicting its local tumor expression. Int J Cancer 138:1994-2002

Li H et al (2015a) A cross-sectional study of the cardiovascular effects of welding fumes. PLoS One 10:e0131648

$\mathrm{Li} \mathrm{H}$ et al (2015b) Oxidative stress, telomere shortening, and DNA methylation in relation to low-to-moderate occupational exposure to welding fumes. Environ Mol Mutagen 56:684-693

Marongiu A et al (2016) Are welders more at risk of respiratory infections? Findings from a cross-sectional survey and analysis of medical records in shipyard workers: the WELSHIP project. Thorax 71:601-606

Matrat $\mathrm{M}$ et al (2016) Welding, a risk factor of lung cancer: the ICARE study. Occup Environ Med 73:254-261

O'Shannessy DJ et al (2012) Folate receptor alpha expression in lung cancer: diagnostic and prognostic significance. Oncotarget $3: 414-425$ 
Ramani VC, Purushothaman A, Stewart MD, Thompson CA, Vlodavsky I, Au JLS, Sanderson RD (2013) The heparanase/syndecan-1 axis in cancer: mechanisms and therapies. FEBS J 280:2294-2306

Seidel C et al (2000) Serum syndecan-1: a new independent prognostic marker in multiple myeloma. Blood 95:388-392

Shen $\mathrm{S}$ et al (2018) Welding fume exposure is associated with inflammation: a global metabolomics profiling study. Environ Health $17: 68$

Siew SS, Kauppinen T, Kyyronen P, Heikkila P, Pukkala E (2008) Exposure to iron and welding fumes and the risk of lung cancer. Scand J Work Environ Health 34:444-450

Sjögren B (2013) Kunskapssammanställning. Hälsoeffekter av gaser och partiklar bildade vid svetsning. Rapport 2013:5, Arbetsmiljöverket: Stockholm. (Knowledge compilation - Health effects from gases and particles emitted during welding. Report 2013:5; Swedish Work Environment Authority, Stockholm. In Swedish)

Sorensen AR, Thulstrup AM, Hansen J, Ramlau-Hansen CH, Meersohn A, Skytthe A, Bonde JP (2007) Risk of lung cancer according to mild steel and stainless steel welding. Scand J Work Environ Health 33:379-386

Sugimoto MA, Vago JP, Teixeira MM, Sousa PL (2016) Annexin A1 and the resolution of inflammation: modulation of neutrophil recruitment, apoptosis, and clearance. J Immunol Res 2016:13

Sundkvist A, Myte R, Boden S, Enroth S, Gyllensten U, Harlid S, van Guelpen B (2018) Targeted plasma proteomics identifies a novel, robust association between cornulin and Swedish moist snuff. Sci Rep 8:2320

Szatmari T, Otvos R, Hjerpe A, Dobra K (2015) Syndecan-1 in cancer: implications for cell signaling, differentiation, and prognostication. Dis Markers 2015:13

't Mannetje A et al (2012) Welding and lung cancer in Central and Eastern Europe and the United Kingdom. Am J Epidemiol 175:706-714

Wang Z, Neuburg D, Li C, Su L, Kim JY, Chen JC, Christiani DC (2005) Global gene expression profiling in whole-blood samples from individuals exposed to metal fumes. Environ Health Perspect 113:233-241

Wong SL, Sukkar MB (2017) The SPARC protein: an overview of its role in lung cancer and pulmonary fibrosis and its potential role in chronic airways disease. Br J Pharmacol 174:3-14

$\mathrm{Xu} J$ et al (2019) SPARC correlates with unfavorable outcome and promotes tumor growth in lung squamous cell carcinoma. Exp Mol Pathol 110:104276

Zeidler-Erdely PC, Meighan TG, Erdely A, Battelli LA, Kashon ML, Keane M, Antonini JM (2013) Lung tumor promotion by chromium-containing welding particulate matter in a mouse model Particle and fibre toxicology. Part Fibre Toxicol 10:45

Zhang Y et al (2015) F2RL3 methylation, lung cancer incidence and mortality. Int J Cancer 137:1739-1748

Publisher's Note Springer Nature remains neutral with regard to jurisdictional claims in published maps and institutional affiliations. 